\title{
OBSERVATORIO TURÍSTO: UNA HERRAMIENTA DE GESTIÓN PARA EL TURISMO DE SOL Y PLAYA EN LA PROVINCIA DE SANTA ELENA
}

Efrén Mendoza, Jhony Yumisaca, Mercedes Freire, Narcisa Ullauri.

Recibido: septiembre de 2016

Aprobado: diciembre de 2016 


\title{
OBSERVATORIO TURÍSTICO: UNA HERRAMIENTA DE GESTIÓN PARA EL TURISMO DE SOL Y PLAYA EN LA PROVINCIA DE SANTA ELENA
}

\author{
TOURIST OBSERVATORY: A MANAGEMENT TOOL FOR SUN \\ AND BEACH TOURISM IN THE PROVINCE OF SANTA ELENA
}

\author{
Efrén Mendoza Tarabó ${ }^{1}$; Jhony Yumisaca Tuquinga ${ }^{1 ;}$ Mercedes Freire Rendón ${ }^{1 ;}$ Narcisa Ullauri Donoso ${ }^{2}$ \\ ${ }^{1}$ Universidad Estatal Península de Santa Elena; emendoza@ upse.edu.ec \\ ${ }^{2}$ Universidad del Azuay; nullauri@uazuay.edu.ec
}

\begin{abstract}
Resumen
El turismo en la provincia de Santa Elena se ha convertido en un factor de gran relevancia apoyado no solo por las políticas gubernamentales, sino también con iniciativas por parte de empresarios y comunidades. La planificación turística se consolida como la mejor herramienta para el desarrollo acertado y sostenible de la actividad apuntando hacia los objetivos establecidos en el Plan Nacional del Buen Vivir, siguiendo los lineamientos del PLANDETUR 2020, y acogiendo a la vez las recomendaciones basadas en larealidad donde el turismo surge. Una de las necesidades evidentes se fundamenta en la carencia de información confiable disponible acerca de las características de la demanda respecto a la oferta del turismo de sol y playa, producto estrella de la provincia, datos que respalden la toma de decisiones pertinentes para cada uno de los actores que integran este amplio sector. El presente artículo muestra algunos resultados alcanzados dentro dela investigación con la aplicación de acciones emprendidas para el diseño de un observatorio turístico direccionado al turismo de sol y playa en Santa Elena, monitoreadas a través del establecimiento de una línea base de directrices de un sistema de gestión de información turística, y del establecimiento del perfil delademanda nacional.
\end{abstract}

Palabras Clave: Observatorio turístico, Turismo de Sol y playa, demanda nacional

\begin{abstract}
Tourism in the province of Santa Elena has become a sector of great importance, which has been not only supported by government policies, but also by initiatives of businessmen and communities. Tourism planning has become the best tool for successful and sustainable development of the activity aiming towards the objectives set in the National Plan for Good Living, by following the guidelines established by PLANDETUR (Strategic Development Plan for Sustainable Tourism in Ecuador) 2020, while accepting at the same time, the recommendations based on reality where tourism arises. One of the tangible needs is based on the lack of reliable information available about the characteristics of the demand in respect to the Sun and Beach tourism offer; which is the leading product of the province. This data is significant to support decisionmaking for each of the actors that make up this vast sector. This paper shows some results obtained in the investigation through the application of actions taken to design a tourist observatory targeted to the Sun and Beach tourism in Santa Elena, and monitored through the establishment of a base for the guidelines of a management system for tourism information, and by the establishment of the profile of domestic demand.
\end{abstract}

Keywords: Tourist Observatory, Sun and Beach Tourism, Domestic Demand

Recibido: noviembre de 2016

Aprobado diciembre de 2016 


\section{INTRODUCCIÓN}

El turismo es una actividad que incide directamente en los ámbitos económico, social y ambiental, repercutiendo favorablemente en el desarrollo de los países, razón por la cual se ha constituido como el eje dinamizador de economías a escala mundial, basando siempre su progreso en la participación estratégica y oportuna de los sectores público, privado y comunitario. Para el alcance del máximo avance en la industria turística es necesaria la planificación constante direccionada hacia la sostenibilidad de los recursos empleados, la misma que debe fundamentarse en información estadística de primera mano coadyuvando a la toma de decisiones acertadas en base a las tendencias del mercado, los requerimientos de la demanda, así como también a la satisfacción de las expectativas que desean experimentar los visitantes de un destino turístico.

Los observatorios turísticos se constituyen en un proyecto del Ministerio de Turismo del Ecuador, que pretende "periódicamente obtener indicadores prácticos del comportamiento turístico en las provincias ecuatorianas, por ejemplo, número de visitantes por períodos, eventos especiales, ocupación de establecimientos de alojamiento, gasto promedio, evolución del turismo sostenible, entre

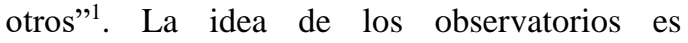
convertirlos en una red nacional a través de un sistema que "podrá dar seguimiento a la evolución del turismo sostenible en los destinos y a nivel nacional, pues deberán tener parámetros homogéneos de aplicación".

Por otra parte, PLANDETUR 2020 orienta a que se debería llevar a cabo el seguimiento e información sobre líneas de productos (ecoturismo, turismo comunitario, MICE, etc.) con la finalidad de permitir una actualización continua de los planes gremiales (ASEC, FENACAPTUR, FEPTCE, etc.) y, de conocer el impacto, la contribución, el grado de sostenibilidad de los proveedores, e información para el desarrollo de nuevos productos.

En este sentido, la presente investigación tiene como objetivo proponer un diseñode Observatorio Turístico en la provincia de Santa Elena para el turismo de sol y playa a través de la coordinación entre la Dirección Provincial de Turismo y la Universidad Estatal Península de Santa Elena, que permita el direccionamiento sostenible de su planificación y toma de decisiones.

El diseño de un observatorio turístico en la Provincia de Santa Elena facilitará la obtención de datos relevantes, permitiendo consolidar el desarrollo de la actividad en este destino, estableciendo un espacio que genera investigación continua y pertinente de este fenómeno social, intercambio de experiencias en la relación oferta-demanda, y que se complementa con la publicación de boletines informativos de los resultados obtenidos en su accionar.

La investigación se centra en el estudio efectuado en cinco balnearios de uso tradicional (mayor afluencia) en la zona de estudio, con lo cual se busca alcanzar los siguientes objetivos específicos:

a. Establecer la línea Base de Investigación para el Observatorio Turístico.

b. Diseñar un sistema de gestión de información turística basada en la oferta y la demanda.

c. Establecer el perfil de la demanda nacional basado en la oferta de sol y playa de la provincia de Santa Elena.

El accionar de un observatorio de este tipo debe poseer un equipo técnico de trabajo multidisciplinario y multisectorial, con atención permanente durante todo el año, principalmente en los períodos de mayor demanda respecto a la modalidad de sol y playa, de esta manera se da respuesta a la hipótesis planteada ¿el diseño de un observatorio turístico en la provincia de Santa Elena permitirá una adecuada planificación en el turismo de sol y playa?

\section{2.- Marco conceptual}

La provincia de Santa Elena, pese a estar considerada entre las primeras cinco que presentan mayor concentración de la demanda extranjera a nivel país y ocupar el tercer lugar de aquellas que concentra la mayor demanda nacional $^{2}$, no cuenta con datos propios $\mathrm{y}$ confiables que permitan tomar decisiones trascendentales, desarrollar propuestas de investigación, monitoreo y evaluación respecto al accionar de la actividad, motivo por el que, la gestión y planificación turística en la modalidad de sol y playa, se ve limitada en la programación de nuevas acciones que propendan a mejorar el posicionamiento de este destino.

El Plan Nacional del Buen Vivir identifica los problemas y necesidades que aborda el turismo en la matriz de tensiones y problemas de la Zona 5 a la que pertenece la provincia de Santa Elena, señalando dentro del contexto de "Economía Social y Solidaria" al objetivo 8 que se refiere a "Consolidar el sistema económico social y solidario, de forma sostenible", especificando en el eje de Matriz Productiva el "Turismo de la 
Ruta Spondylus, Galápagos y temático", el mismo que se relaciona directamente con el accionar de la actividad objeto de estudio en la provincia en cuanto al producto estrella de sol y playa. Así también se relaciona con el objetivo 10 que hace referencia "Al impulso a la transformación de la matriz productiva", cuya meta a priorizar es el incremento "en los ingresos por turismo sobre las exportaciones de servicios totales", enfatizando sus esfuerzos en el ecoturismo, turismo cultural, comunitario, convencional, de playa, montaña y aventura, que tributa a construir espacios de encuentro común $\mathrm{y}$ fortalecer la identidad nacional, las identidades diversas, la plurinacionalidad y la interculturalidad, impulsando la transformación de la matriz productiva.

La idea de los observatorios turísticos es relativamente nueva en el país; solamente se tienen algunas experiencias que publica la página web del Ministerio de Turismo en cuanto a Galápagos, así como iniciativas de universidades como el caso de la Universidad Estatal Amazónica en la ciudad de Puyo; la Universidad Tecnológica Equinoccial en Quito y los estudios realizados por la Universidad Estatal Península de Santa Elena respecto a afluencia turística durante la temporada vacacional de la costa ecuatoriana (enero - mayo).

Es importante señalar que en el ámbito internacional las experiencias sobre modelos de observatorios turísticos es amplia, existen casos en México y España que han sido creados y desarrollados para aportar no solamente datos reales que le proporcionen al sector empresarial y académico herramientas para la toma de decisiones y procesos de planificación, sino que además se ha buscado facilitar información para todos los agentes del turismo, así como también la apertura de diálogos y debates con el fin de analizar y consensuar aspectos relevantes en cuanto al futuro de la actividad, motivo por el cual no debe considerarse el diseño de un observatorio como algo de moda en el país, ni tampoco una instancia limitada de información.

Los observatorios turísticos "son sistemas integrados de información, estudio, investigación y seguimiento del turismo en un destino"3, que se convierten en una herramienta fundamental para la planificación turística, la toma de decisiones oportunas y el mejoramiento de la competitividad del sitio de estudio, teniendo como base la información estadística de primera mano, levantada a través de la oferta y la demanda. Así también Torres ${ }^{4}$ señala que "es un instrumento de investigación científica y de orientación técnica en cuanto a actividades turísticas, el mismo surge como respuesta a la carencia de información eficaz, veraz y oportuna para una mejor toma de decisiones por parte de los actores del turismo, así como la ausencia de investigaciones científicas en el sector turístico" , descripción que coincide con la realidad actual de la provincia de Santa Elena que muestra como una debilidad la falta de datos estadísticos que sirvan de referencia para una planificación adecuada que se sustente en información levantada oportunamente, que permita además de una coordinación con las instituciones vinculadas a la actividad, la socialización de los resultados en todos los estamentos del turismo en todos los niveles.

En el trabajo realizado por Alvarado ${ }^{5}$ se realiza un enfoque a la gestión para la conformación de un observatorio turístico, especificando que "los procesos principales del observatorio obedecen a las demandas de los actores del turismo, los cuales se concretan en las tres categorías identificadas como relevantes o significativas (información, investigación y gestión). Por consiguiente, la oferta de la que esos mismos actores son beneficiarios se concreta en productos o servicios del portafolio del Observatorio. Esa respuesta, debe evaluarse permanentemente para verificar su pertinencia y para ajustar apropiadamente las ofertas a las demandas de conformidad con el concepto de retroalimentación". Esta apreciación muestra la importancia de la participación de cada uno de los integrantes del accionar turístico (sector público, privado y comunitario), situación que permitiría partir de sus requerimientos para el levantamiento de información, la orientación respecto a las líneas de investigación en las que se debe basar su desarrollo y el cumplimiento en la solución de los problemas y necesidades planteadas para su posterior socialización.

El documento de diseño e implementación de un observatorio turístico en los Destinos de Manzanillo y Comala, presentado en el Congreso de Investigación Turística en la Universidad de Colima, México (2012) propone la orientación del observatorio, señalando que este "no busca entrar en colisión con otras instituciones y organizaciones que generan estadísticas de turismo, por el contrario persigue la complementariedad y coordinación con otras actuaciones de índole oficial que puedan desarrollarse para promocionar los destinos e impulsar su desarrollo y la profesionalización del sector turístico", lo que permite orientar los lineamientos para el establecimiento del sistema de gestión de información turística, al abordar los aspectos relacionados a lo que busca medir el observatorio en una región en particular sin 
competir o duplicar esfuerzos con las acciones que puedan emprender el Ministerio de Turismo, así como los Gobiernos Autónomos Descentralizados a nivel provincial y cantonal, estamentos que al final serán parte imprescindible en el análisis de los datos que puedan resultar de los estudios levantados por el observatorio para la toma de decisiones.

El Plan Nacional del Buen Vivir ${ }^{6}$ 2013-2017 menciona que "El cambio de la matriz productiva debe asentarse en el impulso a los sectores estratégicos, en la redefinición de la composición de la oferta de bienes y servicios, orientada hacia la diversificación productiva basada en la incorporación de valor agregado, en el impulso a las exportaciones y su expansión en productos y destinos", el turismo como uno de los sectores identificados debe ser desarrollado a partir de las fortalezas que presenta cada región, Santa Elena requiere aportar con la diversificación de sus productos, para lo cual es necesario partir del estudio encaminado a identificar la situación actual y potencial como destino de sol y playa reconocido a nivel nacional e internacional. El diseño del observatorio turístico contribuirá a una planificación acorde a lo esperado en el PNBV con el fortalecimiento de esta actividad.

Bartolomé Marco $^{7}$ en su Metodología para la creación y desarrollo de un Observatorio Turístico de la provincia de Alicante (España), enfoca las principales razones por las que es imperiosa la necesidad de contar con información confiable respecto a la oferta y demanda del sitio objeto de estudio con miras a incrementar el nivel de satisfacción de los turistas basados en sus requerimientos especificando que "no queremos limitarnos simplemente a ofrecer información útil y agregada a las empresas del sector, ya que el observatorio que tratamos de poner en marcha tiene la característica de ser un proceso de investigación y de aprendizaje abierto, que conlleva necesariamente reflexión y que cuenta en todo momento con la colaboración de los principales destinatarios de los resultados", siendo importante señalar que la propuesta de este diseño plantea su ubicación en la Universidad Península de Santa Elena contribuyendo a la formación profesional de los futuros licenciados en turismo como parte del proceso de enseñanza aprendizaje que la sociedad demanda en los actuales momentos, generando, además de una investigación constante, una vinculación directa con los actores turísticos públicos, privados y comunitarios.

Hernán Gabriel $^{8}$ en su artículo la importancia de un observatorio turístico en Provincia de Buenos Aires señala que "Es importante destacar que un observatorio que se implementa a nivel provincial debe cumplir con un conjunto de funciones relacionadas con el gobierno de ese territorio, por lo tanto los objetivos del observatorio deben estar también en sintonía con la planificación que lleve adelante el gobierno provincial como instrumento de orientación en la toma de decisiones y en la planificación", documento que resalta la relevancia de la coordinación continua y permanente entre los actores del turismo, de allí el involucramiento directo en la toma de decisiones y orientaciones para el diseño del mismo, en este caso la participación de representantes de la anteriormente denominada Dirección Provincial de Turismo de Santa Elena, ahora Oficina de Gestión y Promoción Turística, ha sido pertinente para la presente propuesta, generando un compromiso para planificar adecuadamente el turismo en la zona de estudio a la vez que se delinean algunas de las funciones de este instrumento de gestión.

El Proyecto de observatorio de turismo para Medellín y Antioquia de López y Márquez ${ }^{9}$ plantea "identificar los aspectos de mayor relevancia para los gestores y los usuarios de los observatorios analizados, además de identificar los factores clave de éxito de cada observatorio, con el fin de capitalizar las experiencias en la propuesta presente" a partir del análisis de los observatorios turísticos en el mundo, situación que se fortalece en este documento considerando la revisión de experiencias de países que han diseñado la conformación de sus propios laboratorios como el caso de Colombia, Argentina, México y España, lo cual tributa en este estudio para dar un enfoque de pertinencia, competitividad y alcance de las dependencias expuestas, planteando a su vez los sectores que formarían parte para la captación de datos confiables, así como también los lineamientos para implementar esta herramienta de gestión.

Finalmente, debe resaltarse que, si bien existen artículos referentes a los observatorios turísticos, estos han contribuido al presente trabajo gracias al análisis de las experiencias vividas en diferentes lugares del mundo que han trazado dentro de sus objetivos la creación e implementación de esta herramienta de gestión para mejorar el turismo desde cada uno de sus destinos.

\section{MATERIALES Y MÉTODOS}

El observatorio turístico propuesto tiene como zona de estudio la provincia de Santa Elena $\left(3,762.8 \mathrm{~km}^{2}\right.$.), la misma que incluye tres cantones Salinas, La Libertad y Santa Elena (capital), y se prevé su ubicación estratégica en 
la Universidad Estatal Península de Santa Elena, como centro de estudios superiores.

Para el desarrollo de este trabajo se tomaron como referencia los balnearios turísticos de mayor afluencia en la provincia de Santa Elena: San Lorenzo y Chipipe en Salinas; Ballenita, San Pablo, Ayangue y Montañita, en Santa Elena, los datos estudiados corresponden al estudio de capacidad de carga (2012) de estos sitios, cuyas encuestas aplicadas a los visitantes permitiendo el desarrollo de uno de los objetivos específicos estipulados, tal es el caso del perfil del turista de sol y playa, información de relevancia dentro de los observatorios turísticos

La muestra de servidores turísticos con la que se trabajó procede de la información proporcionada por el Departamento de Turismo de Salinas (Catastro 2010) y la Dirección Provincial de Turismo de Santa Elena (2011), que contribuyeron a determinar los establecimientos registrados en estas entidades.

Tabla 1 Servidores turísticos en los sitios de estudio.

\begin{tabular}{|l|l|l|}
\hline \multicolumn{1}{|c|}{ LUGAR } & \# SERVIDORES & \multicolumn{1}{c|}{ FUENTE } \\
\hline $\begin{array}{l}\text { San Lorenzo - } \\
\text { Chipipe }\end{array}$ & $\begin{array}{l}184 \text { (78 Aloj.- 106 } \\
\text { Alim.) }\end{array}$ & $\begin{array}{l}\text { Dpto. Turismo } \\
\text { Salinas Catastro } \\
2010\end{array}$ \\
\hline Ballenita & $\begin{array}{l}40 \text { (7 Aloj. - 33 } \\
\text { Alim.) }\end{array}$ & $\begin{array}{l}\text { Actualización CCT - } \\
\text { UPSE 2009 }\end{array}$ \\
\hline San pablo & $\begin{array}{l}50 \text { (1 Aloj. - 49 } \\
\text { Alim.) }\end{array}$ & $\begin{array}{l}\text { Actualización CCT - } \\
\text { UPSE 2009 }\end{array}$ \\
\hline Ayangue & $\begin{array}{l}67 \text { (7 Aloj. - 60 } \\
\text { Alim.) }\end{array}$ & $\begin{array}{l}\text { Actualización CCT - } \\
\text { UPSE 2009 }\end{array}$ \\
\hline Montañita & $\begin{array}{l}142 \text { (92 Aloj. - 50 } \\
\text { Alim.) }\end{array}$ & $\begin{array}{l}\text { Actualización CCT - } \\
\text { UPSE 2009 }\end{array}$ \\
\hline TOTAL & $\begin{array}{l}483 \text { establecimientos (185 alojam. - 298 } \\
\text { alim.) }\end{array}$ \\
\hline
\end{tabular}

Fuente: UPSE 2009 - Dpto. Turismo Salinas 2010

Mientras tanto, para la selección de la muestra de los turistas, se consideraron los datos proporcionados por la UPSE relacionados con el monitoreo de afluencia turística registrada durante el carnaval 2012 en los balnearios identificados en la zona de estudio, generando un universo de 204.591 personas.

Tabla 2 Afluencia turística carnaval 2012 sitios de estudio

\begin{tabular}{|c|c|}
\hline LUGAR & \# TURISTAS \\
\hline San Lorenzo - Chipipe & 104.006 \\
\hline Ballenita & 10.715 \\
\hline San Pablo & 29.765 \\
\hline Ayangue & 28.582 \\
\hline Montañita & 31.523 \\
\hline TOTAL & $\mathbf{2 0 4 . 5 9 1}$ \\
\hline
\end{tabular}

Fuente: UPSE 2012

El método utilizado en el muestreo para ambos casos fue el probabilístico estratificado, lo que significó que el universo estuvo dividido considerando los subgrupos homogéneos de acuerdo a los lugares, existiendo un total de cincositios empleados para la investigación, los cuales mantuvieron un orden de localización desde Salinas hasta el norte de Santa Elena. El mecanismo de sorteo aplicado fue el proporcionado a través del programa STATS, obteniéndose de esta manera la muestra, que en el caso de los servidores turísticos resultó en 219 encuestas, mientras que en los turistas llegó al resultado de 399, valor al que se aplicó la fórmula de la fracción muestral $(\mathrm{n} / \mathrm{N})$ para identificar la proporción que se ejecutaría en cada balneario sitio de estudio. En base a lo especificado se detalla el procedimiento del muestreo estratificado:

1. El criterio de estratificación de los balnearios y servidores se determinó por cada lugar de estudio.

2. El registro de los establecimientos y la afluencia de turistas que integran cada uno de las 5 playas mantiene un orden de localización desde Salinas hasta el norte de Santa Elena.

3. Se calculó el tamaño muestral (n):

$$
n=\frac{\mathrm{N}}{\mathrm{e}^{2}(\mathrm{~N}-1)+1}
$$

a) Servidores turísticos

$$
\begin{gathered}
n=\frac{483}{0,05^{2}(483-1)+1} \\
n=\frac{483}{0,0025(482)+1} \\
n=\frac{483}{2,205} \\
n=219,04
\end{gathered}
$$

Tabla 3 Muestra de servidores turísticos.

\begin{tabular}{|l|c|c|}
\hline \multicolumn{1}{|c|}{ BALNEARIO } & $\begin{array}{c}\text { ELEMENTO } \\
\text { x FRACCIÓN }\end{array}$ & $\begin{array}{c}\mathbf{N}^{\circ} \mathbf{A} \\
\text { APLICAR }\end{array}$ \\
\hline San Lorenzo - Chipipe & $184 \times 0,4534$ & 84 \\
\hline Ballenita & $40 \times 0,4534$ & 18 \\
\hline San Pablo & $50 \times 0,4534$ & 23 \\
\hline Ayangue & $67 \times 0,4534$ & 30 \\
\hline Montañita & $142 \times 0,4534$ & 64 \\
\hline \multicolumn{2}{|c|}{ TOTAL } & $\mathbf{2 1 9}$ \\
\hline
\end{tabular}

Fuente: UPSE 2009 - Dpto. Turismo Salinas 2010

b) Turistas

$$
\begin{gathered}
\frac{204591}{0,02^{2}(204591-1)+1} \\
n=\frac{204591}{0,0004(204590)+1} \\
n=\frac{204591}{82,836} \\
n=2469,83
\end{gathered}
$$


Tabla 4 Muestra de turistas

\begin{tabular}{|c|c|c|}
\hline ESTRATO & ELEMENTO $\times$ f & CUPO \\
\hline $\begin{array}{ll}\text { San Lorenzo - } \\
\text { Chipipe }\end{array}$ & $\begin{array}{l}104006 \mathrm{x} \\
0,012073\end{array}$ & 1256 \\
\hline Ballenita & $10715 \times 0,012073$ & 129 \\
\hline San Pablo & $29765 \times 0,012073$ & 359 \\
\hline Ayangue & $28582 \times 0,012073$ & 345 \\
\hline Montañita & $31523 \times 0,012073$ & 381 \\
\hline \multicolumn{2}{|c|}{ TOTAL } & 2470 \\
\hline
\end{tabular}

Fuente: UPSE 2012

Se empleó el método inductivo-deductivo y bibliográfico, se revisaron y analizaron artículos publicados en la web relacionados con los observatorios turísticos, para la realización de los gráficos e interpretación de los resultados obtenidos se emplearon tablas de dinámicas (Excel). Adicionalmente se aplicaron entrevistas a 6 actores del desarrollo turístico de la Provincia de Santa Elena (muestra no probabilística por expertos) para conocer su criterio respecto a la necesidad de contar con un observatorio turístico y los lineamientos que deberían incorporarse. Entre quienes colaboraron constan los representantes de la Dirección Provincial de Turismo, Dirección Municipal de Turismo de Salinas, Empresa Municipal de Turismo de Santa Elena, Gobierno Provincial de Santa Elena, Representante del gremio hotelero y el Director de la Escuela de Hotelería y Turismo de la UPSE.

\section{RESULTADOS}

El diseño de un observatorio turístico en la provincia de Santa Elena para el turismo de sol y playa busca fortalecer y orientar el desarrollo de la actividad en el área urbana y rural donde se encuentran los principales balnearios que gozan de la mayor demanda, basando su accionar en el avance sostenible y sustentable de sus recursos naturales y culturales, así como también con la participación de las entidades vinculadas al turismo a nivel provincial y cantonal, con el aporte de los representantes comunales y los servidores turísticos, creando mesas de diálogo y concertación que deriven en soluciones a las necesidades y problemas existentes como consecuencia de la afluencia en las diferentes épocas de visita.

De la misma manera el observatorio se convierte en el instrumento de investigación que, sustentado en un equipo técnico, direcciona su accionar en beneficio del patrimonio santaelenense, buscando el desarrollo potencial y sostenido del recurso playa, contribuyendo a E. Mendoza/J. Yumisaca/M. Freire/N. Ullauri elevar el nivel de conocimiento del comportamiento de la demanda para dar valor agregado a su experiencia, elevando el nivel de satisfacción de los ofertantes y los demandantes.

El sector económico vinculado al turismo (alojamiento, alimentación, intermediario y recreación en sus diversas modalidades micro, pequeña y mediana empresa) presentará su desarrollo al momento de proveer a los administradores información estadística veraz y actualizada respecto a la realidad provincial, que les permita proyectar sus acciones en base a los estudios sugeridos por ellos para la toma de decisiones. La ejecución oportuna de la necesidad de la oferta y demanda permitirá mejorar los ingresos y orientar su promoción hacia segmentos más especializados en el mercado interno y externo.

El diseño del observatorio turístico a nivel provincial, considerando la experiencia de otros países y aquellos planteamientos a nivel nacional solucionarían directamente las principales necesidad planteadas en la mesa de concertación con el SENESCYT y los actores de la península (base de datos estadísticos, diseño de productos turísticos sostenibles alternativos, capacitación, impacto generado por el turismo tradicional e impacto generado por el cambio de la modalidad educativa por quimestres en escuelas y colegios en el país).

Es relevante señalar que el Ecuador no cuenta en la actualidad con una Cuenta Satélite de Turismo (CST), que de acuerdo a la Organización Mundial del Turismo OMT ${ }^{10}$ se convierte en "un marco estadístico normalizado y el principal instrumento de medición económica del turismo" $\mathrm{y}$, plantea en su documento Recomendaciones internacionales para estadísticas de turismo, publicado en el año 2010 junto a las Naciones Unidas, en su capítulo uno sobre desarrollo y necesidad de estadísticas.

Estas recomendaciones tienen como objetivo "proporcionar un sistema de conceptos, definiciones, clasificaciones e indicadores que sean coherentes a nivel interno, y que faciliten el vínculo con los marcos conceptuales de la Cuenta Satélite de Turismo, los sistemas de cuentas nacionales, la balanza de pagos y las estadísticas de trabajo, entre otros"

Ante esta situación, el observatorio turístico de Santa Elena se establece como el instrumento idóneo para recuperar esta fuente de datos inexistentes en la provincia, el mismo que, al estar en estrecha coordinación con los representantes del Ministerio de Turismo a través 
de la Oficina de Gestión y Promoción Turística, permitirá lo que la OMT enfatiza, "la armonización y la conciliación de las estadísticas de turismo desde una perspectiva económica", contribuyendo de manera significativa en la generación de datos económicos confiables que podrían ser comparados con información adicional que revele el comportamiento y la tendencia de este importante sector que forma parte de la matriz productiva del país.

Santa Elena ha demostrado ser un destino posicionado en el mercado nacional e internacional, preferido por las características variadas a lo largo de su perfil costero, sus estamentos públicos, privados y comunitarios relacionados con el sector requieren de una pronta intervención en el desarrollo del turismo que conlleve a una oportuna planificación sustentada en datos creíbles que resulten de estudios académicos validados y que respondan a dichas exigencias, fortaleciendo a su vez las iniciativas propuestas por estos actores en el poco operativo y casi desaparecido en la actualidad, Comité Técnico de Turismo de Santa Elena que agrupa a los representantes de los Gobiernos Autónomos Descentralizados de la Provincia, Municipios de Salinas, La Libertad y Santa Elena, Parroquiales, Comunitarios y Empresarial.

Por su parte el Plan Estratégico de Desarrollo Sostenible para el Ecuador - PLANDETUR 2020, plantea en su programa Sistema de Información Estratégica para el Turismo del Ecuador (SIETE) que "una de las herramientas más importantes para una gestión planificada en general y especialmente en el campo del turismo es la información, la que debe estar disponible para todos los usos que se pueden hacer de ella, especialmente la toma de decisiones estratégicas en situaciones de crisis, la investigación, el monitoreo y la evaluación del sector", para lo cual propone el proyecto la creación de un Sistema de Observatorios Turísticos por Destino, que se alinea directamente a la propuesta planteada y que busca la "Creación de un Sistema de Observatorios Turísticos Provinciales que otorguen periódicamente indicadores prácticos del comportamiento turístico en las provincias ecuatorianas, (por ejemplo, número de visitantes por períodos, eventos especiales, ocupación de establecimientos de alojamiento, gasto promedio, evolución del turismo sostenible, etc.)".

El diseño del observatorio en Santa Elena se convierte en una herramienta que aporta de manera beneficiosa al desarrollo turístico del país, con la creación y administración adecuada de este laboratorio turístico de investigación. Por lo tanto, se establece la respectiva misión y visión del observatorio:

Misión: Proporcionar una base de datos pertinente, actualizada y consensuada, relacionada al turismo de sol y playa de la Provincia de Santa Elena a los diferentes actores del sector público, privado y comunitario, así como a los diferentes usuarios, mediante el uso de herramientas y tecnología de primera en el levantamiento de información que permita la toma de decisiones adecuadas en los aspectos de la planificación sostenible y sustentable del recurso natural y cultural inmerso en el producto ofertado.

Visión: El observatorio turístico de sol y playa de la provincia de Santa Elena es el principal referente confiable de información relacionada con el producto estrella de la zona, el mismo que en base a los resultados generados de las investigaciones brinda los lineamientos oportunos para el desarrollo sostenible del turismo de manera consensuada con los diferentes actores provinciales.

Considerando los lineamientos antes mencionados se establecen las siguientes funciones del observatorio turístico:

i) Diseño, validación y aplicación de los instrumentos (encuestas, entrevistas, fichas de observación directa) para el levantamiento de información actualizada de la realidad del turismo de sol y playa en la provincia de Santa Elena como parte del diagnóstico situacional que permitirá proyectar y comparar su evolución a través del seguimiento y monitoreo.

ii) Circunscripción de la población que formará parte del objeto de estudio, así como la selección de la muestra a encuestar o entrevistar basados en lineamientos claramente identificados y consensuados con los actores del turismo.

iii) Planificación de sesiones de trabajo con los agentes del desarrollo del sector turístico público, privado y comunitario para el establecimiento de las líneas de acción del observatorio partiendo de la identificación del comportamiento de la demanda, sus necesidades, expectativas, tendencias y evaluación de los servicios ofertados.

iv) Diseño y ejecución de estudios de mercado; benchmarking; elaboración y comercialización de la marca del destino; y 
demás estudios que plantee el consenso de los diferentes actores del turismo.

v) Elaboración del plan anual del observatorio mediante la discusión, análisis y toma de decisiones en coordinación con los representantes del sector público, privado y comunitario involucrados en el desarrollo del turismo.

vi) Difusión de los resultados respecto al desarrollo del turismo, su proyección y planificación para toda la población de Santa Elena, medios de comunicación y comunidad académica del sector y de la región mediante la elaboración de informes o boletines mensuales y anuales.

vii)Ejecución de eventos de debates, foros, y otros que conformen puntos de encuentro entre los sectores públicos, privados y comunitarios con enfoque académico que tribute a la generación de espacios de formación.

viii) Diseño y publicación de artículos de carácter científico en revistas indexadas y especializadas que permitan el conocimiento ante la sociedad de los principales resultados producto de las investigaciones aplicadas.

ix) Celebración de convenios marcos y específicos con instituciones públicas, privadas y comunitarias como parte de las alianzas estratégicas que contribuirán al mantenimiento del observatorio turístico.

x) Promoción de famtrips (viajes de familiarización) y presstrips (viajes para la prensa) para el logro del posicionamiento de los diferentes balnearios que oferta la provincia, fortaleciendo de esta manera la promoción de los destinos.

xi) Diversificación de la oferta gastronómica de la provincia, a través del diseño de un producto que permita la recuperación de platos basados en la riqueza ictiológica del sector, así como también el posicionamiento de nuevos platos, propuesta que se complementaría directamente con el turismo de sol y playa sin dejar que disminuya su protagonismo como producto estrella.

xii)Planteamiento de políticas turísticas que beneficien el desarrollo del turismo de sol y playa, que además eleve el nivel de satisfacción de los servidores turísticos y de los demandantes del producto ofertado. xiii) Otras que los administradores del observatorio, en base a las necesidades de los actores del turismo, lleguen a consensuar de manera objetiva para el fortalecimiento del laboratorio turístico.

Los actores que formarán parte de la discusión, análisis y toma de decisiones involucra en el sector público a: Oficina de Gestión y Promoción Turística (representación de la Coordinación Zonal cinco del Ministerio de Turismo), Dirección Provincial de Ambiente, Dirección Provincial de Cultura, Direcciones de Turismo de los GAD's Seccional y Local, Comisiones de Turismo de los GAD's de las Juntas Parroquiales y Carreras de Turismo de las Instituciones de Educación Superior representativas de la provincia, en este caso la Universidad Estatal Península de Santa Elena; por su parte los actores del sector privado estaría integrado por representantes de: Asociaciones de Hoteleros, Restaurantes y Cabañas Comedores de la provincia, Representante de las Agencias de Viajes u Operadoras, representantes de fundaciones afincadas en la provincia con proyectos de desarrollo turístico; finalmente los actores del sector comunitario involucraría a Presidentes de comunidades, Comités/Asociaciones de Turismo debidamente legalizadas y representantes de proyectos turísticos comunitarios.

Los actores serán los encargados desde sus instancias de gestionar los recursos que permitan la operatividad del observatorio turístico, situación que asegurará el uso de los resultados de los estudios e investigaciones que se generen en el seno del observatorio para la planificación, local y provincial del turismo.

Por aspectos estratégicos las oficinas para el funcionamiento del observatorio turístico se ubicarán en los predios de la Universidad Estatal Península de Santa Elena (UPSE), basado en los criterios de a) generación de conocimiento, b) presencia de profesionales debidamente capacitados y con experiencia en la región y el país, c) desarrollo de proyectos de investigación y vinculación relacionados con la actividad, d) predisposición de capital humano para las principales acciones y, e) credibilidad en su accionar sin influencia política e intereses personales.

Para este fin, la UPSE debe asignar la responsabilidad a la Dirección de las Carreras de Turismo y Hotelería, quien actuaría como delegado del Rector de la entidad, así como también, debe considerarse el organigrama de esta nueva dependencia para el correcto 
funcionamiento, el mismo que estaría estructurado de la siguiente manera:

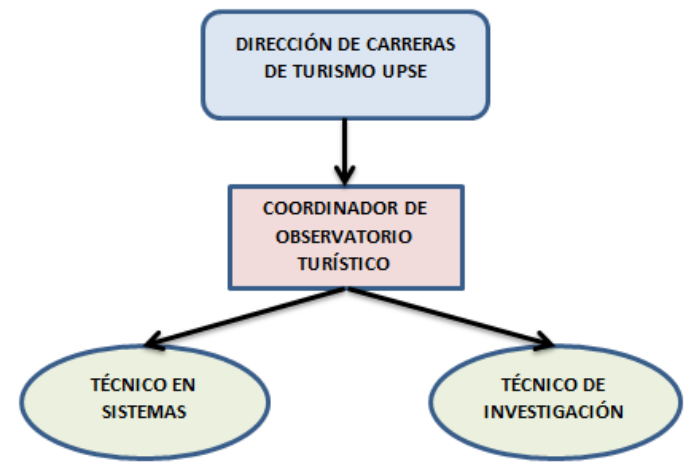

Grafico 1. Estructura Orgánica. Elaborado por autores

El Director de Carreras de Turismo de la Universidad será el profesional encargado de representar, junto al Coordinador al Observatorio Turístico, quienes deberán encargarse de la gestión ante los delegados de las entidades públicas, privadas y comunitarias, generando los espacios de diálogo y concertación; de la misma manera, la planificación de las acciones fundamentadas en las necesidades y requerimientos de los principales actores del desarrollo turístico y de los temas relacionados con las tendencias del mercado, buscando el bienestar de la comunidad y de la academia. Su papel se orienta principalmente a la asesoría.

El Coordinador del Observatorio Turístico deberá cumplir con las siguientes funciones:

1. Representar a la entidad en las sesiones de trabajo relacionadas con el desarrollo de la actividad turístico en la provincia de Santa Elena.

2. Administrar los recursos asignados al observatorio.

3. Planificar anualmente las acciones orientadas a los estudios e investigaciones que ejecutará el observatorio.

4. Presidir las sesiones de trabajo emitiendo las convocatorias respectivas a los actores del sector público, privado, comunitario y académico, para la coordinación pertinente de las acciones programadas.

5. Establecer conjuntamente con los representantes turísticos las políticas que regirán en el manejo y funcionamiento del observatorio.

6. Velar por el cumplimiento de las acciones asignadas a los técnicos (sistemas e investigación) del observatorio.

7. Dirigir el cumplimiento de los estudios y proyectos de investigación previamente establecidos y aprobados.
8. Definir las estrategias a aplicar para el levantamiento de datos requeridos en los diferentes estudios y proyectos.

9. Emitir informes mensuales y de rendición de cuentas semestrales que demuestren los avances y resultados principales de los proyectos en ejecución.

10. Proponer proyectos de investigación de índole turística que tributen al fortalecimiento de la actividad y a la toma de decisiones requeridas por los actores en base a necesidades, expectativas y tendencias de desarrollo.

11. Promover el acercamiento entre los actores para el consenso en las acciones a ejecutar.

12. Difundir los resultados obtenidos de las investigaciones a los actores del turismo y la comunidad en general como parte de la socialización y la vinculación con la colectividad.

13. Establecer convenios marco de cooperación interinstitucional con las diferentes instituciones a nivel nacional y provincial que tributen a los objetivos del observatorio turístico.

El Técnico en Sistemas tendrá las siguientes funciones a su cargo:

1. Establecer las herramientas informáticas que se emplearán en la ejecución de los estudios y proyectos planificados, para lo cual deberá proponer los equipos requeridos para el buen funcionamiento del observatorio.

2. Aplicar las herramientas tecnológicas para plasmar la información levantada en mapas digitales, así como otros elementos requeridos por el observatorio.

3. Participar en cada una de las sesiones de trabajo a las cuales fuere convocado para la planificación de estudios e investigaciones y la presentación de informes cuando se requiera.

4. Diseñar y administrar la base de datos de la información recopilada en los diferentes estudios e investigaciones, así como también de la página web del observatorio turístico.

5. Colaborar coordinadamente en las diferentes acciones para el buen desempeño y operación del observatorio.

El Técnico de Investigación deberá regirse a:

1. Coordinar y ejecutar el levantamiento de información requerida en los distintos estudios e investigaciones con la participación de los estudiantes de las carreras de turismo de la UPSE.

2. Diseñar los formatos $y$ herramientas requeridas para los estudios e investigaciones 
que permitan la recopilación de datos pertinentes.

3. Presentar los resultados de la información levantada en cuadros estadísticos que permitan la adecuada interpretación y la toma de decisiones ante los actores de turismo y comunidad en general cuando sea el caso.

4. Participar en cada una de las sesiones de trabajo a las cuales fuere convocado para la planificación de estudios e investigaciones y la presentación de informes cuando se requiera.

5. Colaborar coordinadamente en las diferentes acciones para el buen desempeño y operación del observatorio.

En los actuales momentos la provincia de Santa Elena presenta algunas tensiones y problemas identificados dentro de la Zona 5 a la cual pertenece, situación que fue abordada en las entrevistas que se realizaron con los actores del turismo, siendo el denominado "Ecoturismo, turismo cultural, comunitario, convencional, de playa, montaña y aventura" el que se relaciona con la investigación desarrollada en lo referente al turismo de sol y playa; así también se destacaron las fortalezas existentes.

Dentro del análisis se coincidió en que el desarrollo del producto estrella de Santa Elena se ha dado en sus inicios de manera espontánea, sin embargo, en los últimos veinte años se evidencia un progreso gracias a las iniciativas de planificación turística desarrolladas por instancias gubernamentales como el Programa de Manejo de Recursos Costeros (PMRC), Oficina Técnica de Turismo, UPSE y Direcciones de Turismo de los GAD's municipales, así como de ONG's que incluyeron en sus agendas de trabajo al turismo, fortaleciendo varias propuestas de turismo en las comunidades asentadas en el norte de la zona de estudio. Así también el aporte del Ministerio del ramo permitió que, como parte del proceso de Descentralización del Turismo, los cantones asumieran varias funciones dentro de la actividad entre los años 2001 (Salinas y Santa Elena) y 2003 (La Libertad).

Para establecer la línea Base de Investigación para el Observatorio Turístico, se consultó a los actores del turismo respecto a qué información debería ser considerada para establecer la línea base de investigación para el observatorio turístico en Santa Elena, partiendo de las necesidades y demandas generadas por ellos, precisándose los siguientes aspectos:

a. Recopilación de información disponible de la oferta y demanda de la provincia, para lo cual se señalaron entre otros documentos existentes: inventario de atractivos turísticos con su respectiva jerarquización; catastro de establecimientos turísticos (alojamiento, alimentación, agenciamiento y recreación); flujo de vehículos reportados por la Comisión de Tránsito del Ecuador en feriados y temporada vacacional de la costa ecuatoriana; monitoreo del seguimiento a turistas en principales balnearios de Santa Elena realizado por la UPSE en los años 2010, 2011 y 2012.

b. Actualización de los datos relacionados con la oferta y demanda, en este sentido se especificó que la documentación existente, si bien puede utilizarse para generar la línea base, es conveniente que se reemplace con información del presente año, lo que aseguraría un mejor acercamiento a la realidad de la zona. Para este fin la UPSE contaría con el talento humano debidamente preparado para el levantamiento respectivo con la colaboración de las demás dependencias públicas. Esta acción derivará en otras actividades como:

- Elaboración de formatos para actualizar los datos.

- Trabajo de campo.

- Elaboración del documento final con los datos actualizados

- Definición de una marca turística que represente a la provincia de Santa Elena.

En relación al diseño de un sistema de gestión de información turística basada en la oferta y la demanda de sol y playa se estableció el siguiente esquema:

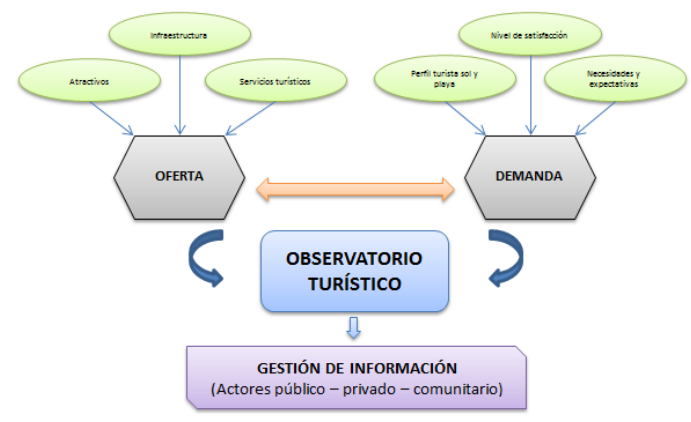

Gráfico2 Sistema de Gestión de Información Turística. Elaborado por el grupo de investigación

El Sistema está conformado por tres elementos claramente identificados: el primer elemento corresponde a la oferta que incluye información relevante respecto a la infraestructura existente, los atractivos actuales y potenciales identificados, así como los servicios turísticos (alojamiento, alimentación e intermediarios); el segundo involucra a la demanda, que requiere la 
incorporación de información relacionada con el perfil del turista de sol y playa; el nivel de satisfacción sobre la oferta, además de las necesidades y expectativas existentes. El tercer elemento corresponde a la gestión de la información en donde se encuentran agrupados los actores del sector público, privado $\mathrm{y}$ comunitario.

El observatorio turístico exige un intercambio constante entre los datos generados por la oferta y la demanda, permitiendo que los actores analicen y evalúen los resultados, a la vez que se generan los bancos de información que tributarán a la toma de decisiones oportunas, esto debe reflejarse en planes de acción debidamente socializados para el desarrollo del turismo de sol $\mathrm{y}$ playa, orientando su mejoramiento $\mathrm{y}$ sostenibilidad.

Respecto al establecimiento del perfil del turista nacional de sol y playa en la provincia de Santa Elena, es importante señalar que la demanda ha variado significativamente en los años 2010, 2011 y 2012, períodos que cuentan con estadísticas de afluencia en este segmento de mercado gracias a la participación de la UPSE con el proyecto de Seguimiento y monitoreo aplicado en varios de los balnearios de Santa Elena, tal como lo muestra la tabla 5. Aquí se refleja el comportamiento de la demanda en los períodos vacacionales de la costa ecuatoriana, específicamente en las 5 playas que son objeto de estudio en la presente investigación.

Tabla 5: Afluencia de turistas de sol y playa en balnearios de estudio Feriado de carnaval

\begin{tabular}{|c|c|c|c|}
\hline BALNEARIO & $\mathbf{2 0 1 0}$ & $\mathbf{2 0 1 1}$ & $\mathbf{2 0 1 2}$ \\
\hline $\begin{array}{c}\text { San Lorenzo - } \\
\text { Chipipe }\end{array}$ & $\begin{array}{c}\text { no } \\
\text { participó }\end{array}$ & 34.046 & 104.006 \\
\hline Ballenita & 5.796 & 8.570 & 10.715 \\
\hline San Pablo & 29.761 & 20.789 & 29.765 \\
\hline Ayangue & 22.715 & 39.933 & 28.582 \\
\hline Montañita & 30.195 & 16.829 & 31.523 \\
\hline TOTAL & $\mathbf{8 8 4 6 7}$ & $\mathbf{1 2 0 1 6 7}$ & $\mathbf{2 0 4 5 9 1}$ \\
\hline
\end{tabular}

Fuente: Seguimiento y monitoreo afluencia de turistas en Santa Elena 2010, 2011 y 2012

Para determinar el perfil del turista se consideraron los últimos datos del año 2012, tabulándose un total de 2470 encuestas que fueron aplicadas en los balnearios de estudio entre el 9 de febrero y el 29 de abril, independientemente de lo establecido en la muestra, obteniéndose los principales resultados con características muy significativas que se presentan en el siguiente resumen:
Tabla 6: Resumen del Perfil del turista de sol y playa de la provincia de Santa Elena

\begin{tabular}{|c|c|}
\hline Variable & Perfil \\
\hline Género encuestado & $\begin{array}{c}51 \% \text { masculino }-49 \% \\
\text { femenino }\end{array}$ \\
\hline Edad & $26-45$ años \\
\hline Procedencia & Guayaquil \\
\hline $\begin{array}{l}\text { Preferencia de } \\
\text { viaje }\end{array}$ & Familia \\
\hline Permanencia & Durante el día \\
\hline Pernoctación & $\begin{array}{c}\text { Establecimientos } \\
\text { hoteleros }\end{array}$ \\
\hline $\begin{array}{c}\text { Información } \\
\text { obtenida }\end{array}$ & Familiares y amigos \\
\hline Motivo de visita & $\begin{array}{l}\text { Recreación o } \\
\text { esparcimiento }\end{array}$ \\
\hline $\begin{array}{l}\text { Gasto promedio } \\
\text { diario }\end{array}$ & $\begin{array}{c}41 \text { - } 50 \text { dólares } \\
\text { americanos }\end{array}$ \\
\hline $\begin{array}{l}\text { Seguridad de la } \\
\text { playa }\end{array}$ & Suficiente \\
\hline
\end{tabular}

Fuente: UPSE, 2012

Si bien existen muchos lineamientos que debe incluirse, es importante reconocer que ahora se cuenta con una descripción base del perfil del turista de sol y playa que se sustenta en una investigación con datos reales que permiten direccionar la toma de decisiones oportunas. El establecimiento del perfil del turista busca proporcionar información especializada respecto a las características de esta demanda, permitiendo la orientación de acciones de marketing para incrementar la afluencia, así como también analizar las necesidades para generar propuestas que eleven la calidad en el nivel de satisfacción al consumir el producto ofertado en la provincia de Santa Elena.

\section{DISCUSIÓN}

En este trabajo se puede apreciar la imperiosa necesidad de contar con un observatorio turístico que canalice la obtención de información de primera mano permitiendo orientar el desarrollo del turismo de sol y playa de una manera sostenible, a la vez que asegura el cuidado del recurso mientras se fortalece el nivel de satisfacción de los turistas al atender las opiniones y expectativas que deben reflejarse en la investigación continua. La toma de decisiones acertada permitirá que el producto estrella de la provincia de Santa Elena se mantenga en una 
etapa de madurez previniendo el declive que generalmente debe cumplirse como parte de su proceso, promoviendo una orientación para alcanzar la certificación turística de las principales playas de acuerdo a la Norma Técnica Ecuatoriana NTE INEN 2631:2012 del Instituto Ecuatoriano de Normalización.

Establecer la línea base de investigación no resultó sencillo, debido a la diversidad de criterios, que de una u otra manera se orientaban a intereses institucionales, válidos en todo sentido, pero que podría relacionarse posteriormente con el accionar político, aspecto que se debió manejar con mucho cuidado para que no se vea afectada en ningún momento la objetividad de la información requerida para el progreso de la zona, mientras que por otro lado el protagonismo para que el observatorio turístico opere sosteniblemente tiene que reflejarse como un solo equipo de trabajo. Las sugerencias sobre las funciones del observatorio fueron analizadas en varios sentidos, permitiendo llegar a consensos para no interferir con algunas de las funciones que asumieron los GAD Municipales dentro del proceso de descentralización.

Entre algunos de los aspectos relevantes de las actividades con los actores deben resaltarse: i) la acción de considerar el trabajo realizado años atrás por varias instituciones públicas, privadas y comunitarias ha sido muy significativo porque valida el progreso continuo del turismo de sol y playa en la zona de estudio; ii) el reconocer que la provincia no cuenta con datos estadísticos importantes para la planificación del turismo y la respectiva toma de decisiones, mientras que los pocos que existen se manejan de forma independiente en cada institución; iii) el interés y la decisión por actualizar la línea base a nivel de provincia como punto de partida del observatorio, lo que permite generar un fundamento en los datos que posteriormente se vayan incorporando para progresivamente ir evaluando el desarrollo del turismo y proyectar su crecimiento.

La estructura del sistema de gestión de información se visualiza como poco complejo, sin embargo, debe cuidarse cada uno de los elementos identificados para que su funcionamiento sea, además de operativo, muy dinámico, que se adapte a las situaciones cambiantes a las que nos tiene acostumbrado el fenómeno del turismo. Los datos que se generen convierten al observatorio turístico en el punto de referencia para el análisis, constante y actualizado del sector, cuyos resultados serán el referente para las instituciones que trabajan en el turismo, entre ellas las de índole académico que contarían con estadísticas reales para la aplicación como herramienta de gestión para la toma de decisiones oportuna, hasta el punto de complementarse con los proyectos de investigación y de vinculación con la colectividad, exigidos en la normativa vigente de educación superior, a la vez que se convierte en una oportunidad para la realización de prácticas pre profesionales.

Estas razones contempladas desde el punto de vista académico son las que sustentan que la ubicación del observatorio se mantenga en la Universidad Península de Santa Elena, una entidad del estado que se fundamenta en el proceso de enseñanza-aprendizaje sin estar necesariamente ligada a un partido político que tienda a buscar intereses propios para su beneficio, situación que fue muy discutida con los actores de los otros sectores públicos, apuntando a que sea considerado como el resultado de la gestión que actualmente lideraba la institución, convirtiéndose en uno de las situaciones de mayor discusión y poco consenso dentro del proceso establecido.

Otro de los aspectos que reflejó diferencias al momento de llegar a acuerdos se identificó con el profesional que estaría dirigiendo el observatorio, debido a que se indicaba que el perfil no debe ser tan exigente en relación a los profesionales existentes en la provincia, sin embargo siempre se fundamentó la necesidad de contar con las características óptimas para desempeñar este cargo considerando que estaría en juego el futuro del turismo a través de la toma de decisiones más acertadas aprovechando su verdadero potencial.

Por su parte el perfil del turista de sol y playa ha permitido romper paradigmas como aquellos relacionados con las estrategias de marketing aplicadas durante años en Santa Elena para la promoción de sus atractivos, es decir, el producto estrella se mantiene pese a los esfuerzos por posicionar el turismo ecológico, comunitario y cultural, lo que indica que debemos ser más innovadores al momento de desarrollar otras alternativas de turismo que se complementen con el principal. La mayoría de encuestados (62\%) señalan que la información proporcionada por familiares y amigos es la más relevante para visitar los balnearios, por encima del internet (13\%) y la televisión (13\%).

Otro de los aspectos necesarios de resaltar es que, si bien el mercado de la ciudad de Guayaquil es el que lidera la demanda en la época invernal, también es representativa la presencia de turistas de provincias como Los Ríos, Manabí y Azuay. 
Además debemos señalar que el $51 \%$ de los encuestados solo permanecen durante el día en los balnearios de estudio, pernoctando solo el $49 \%$, y de ellos el $65 \%$ utiliza establecimientos hoteleros, los demás optan por las casas de familiares y amigos.

En el gasto promedio diario predomina el mayor a US \$ 50,00 dólares americanos con un 34\%, dato que resulta real al comparar con el gasto registrado durante el último feriado del año 2014 que se determinó en US \$70,00 dólares americanos (Investigación de Mercados, MINTUR, 2015).

Los datos del perfil del turista obtenidos se convierten en una información confiable que no existía en la provincia, lo empírico queda relevado ante los resultados que se han presentado, los cuales están debidamente sustentados y respaldados por este trabajo investigativo.

Los resultados de las encuestas tabuladas fueron socializados con los actores involucrados con el turismo en la provincia de Santa Elena, ante lo cual no hubo mayores criterios debido a que era la opinión de quienes disfrutan del sol y playa en los diferentes balnearios; lo que sí se convirtió en un punto fuerte de definir fueron los temas de la línea base de información y el sistema de gestión de información, lo cual llevó un tiempo adicional al considerado, para llegar a un consenso.

Es evidente que la creación del observatorio turístico para el producto de sol y playa en la provincia de Santa Elena centraría toda la información requerida por los diversos sectores para orientar la planificación sostenible del sector, ejerciendo su accionar en cada uno de los susbsistemas del turismo, logrando la participación activa y consensuada de los actores, solucionando los diversos problemas que se presentan continuamente en la toma de decisiones por la inexistencia de datos confiables y actualizados, una razón muy importante para asegurar el futuro de este destino.

\section{CONCLUSIONES}

La presencia de un observatorio turístico para el producto de sol y playa en la provincia de Santa Elena se convierte en una herramienta que apoyaría significativamente el desarrollo del sector debido a la falta de datos estadísticas que permitan tomar acciones correctivas y de promoción que fortalezcan las iniciativas de los diferentes actores públicos, privados $\mathrm{y}$ comunitarios.
El involucramiento del Ministerio del Turismo, a través de la Oficina de Gestión y Promoción Turística de Santa Elena se verá definitivamente con la operatividad de un observatorio.

La participación directa de todos los actores del turismo de Santa Elena identificados en las diferentes sesiones de trabajo permitirá llegar a la toma de decisiones consensuadas y pertinentes que beneficiarán a la oferta y la demanda relacionada con el producto estrella de la provincia, el turismo de sol y playa.

La estructura orgánica del observatorio y el sistema de gestión de información propuesto generan un acercamiento directo de la academia en la ejecución de las diversas acciones a emprenderse para la realización de estudios e investigaciones, permitiendo fortalecer el proceso de enseñanza aprendizaje de los futuros profesionales del turismo.

Se cuenta en los actuales momentos con un perfil básico del turista de sol y playa de Santa Elena.

El observatorio turístico generará resultados y recomendaciones de los diferentes estudios e investigaciones, que tributarán para orientar la planificación que necesita el sector mediante la aplicación de normas y técnicas en pro del bienestar del recurso playa y de la satisfacción de la demanda.

\section{REFERENCIAS BIBLIOGRÁFICAS}

[1]Ministerio de Turismo del Ecuador. Tourism \& Leisure Europraxis Consulting. Plan. Quito. (2007).

[2]Ministerio de Turismo del Ecuador. La Experiencia Turística en el Ecuador. Cifras esenciales de turismo interno y receptor. Dirección de Investigación. Quito (2012).

[3]Conde, E., Covarrubias, R., y Schmidt, N. Diseño e implementación de un observatorio turístico en los Destinos de Manzanillo y Comala. Congreso de Investigación Turística XIII Nacional y VII Internacional de SECTUR/CESTUR. Escuela Superior de Turismo del Instituto Politécnico Nacional. Universidad de Colima. Facultad de Turismo. (2011)

[4]Torres, G. Diseño de un Observatorio Turístico Cultural para la Planificación y Gestión del Turismo Sostenible del Cantón Riobamba. Escuela Superior Politécnica de Chimborazo. Facultad de Recursos Naturales. Escuela de Ingeniería en Ecoturismo. (2012). 
[5] Alvarado, O., Guauña, D., y Rivera, W. Modelo de gestión para la conformación de un observatorio turístico para el departamento del Cauca. Corporación Universitaria Autónoma del Cauca. I Simposio de Investigación. - Capítulo Suroccidente de ASCOLFA. (2010)

[6] SENPLADES, S. N. Buen Vivir Plan Nacional 2013 -2017. Quito. (2013)

[7] García, F., Marco, B., y Sabater, V. Metodología a seguir para la creación y desarrollo de un Observatorio Turístico de la Provincia de Alicante. III Congreso Internacional de Turismo de Leiria y Oeste (Portugal). Dpto. de Organización de Empresas e Instituto Universitario de Investigaciones Turísticas Universidad de Alicante. (2011).

[8] Gabriel, H. La importancia de un observatorio turístico en provincia de Buenos Aires como instrumento de orientación en la toma de decisiones y en la planificación. Notas en Turismo y Economía. Año II. Nro. III. ISSN 1853-1504. (2011)

[9] López, L., y Márquez, J. Proyecto de observatorio de turismo para Medellín y Antioquia. Revista Soluciones de Postgrado EIA, Número 9. p. 79-96. Medellín. (2012).

[10]Naciones Unidas y Organización Mundial del Turismo. Recomendaciones internacionales para estadísticas de turismo, Madrid - Nueva York. (2010).

[11] Estratégico de Desarrollo Sostenible para Ecuador - PLANDETUR 2020. Informe final.

[12] Universidad Estatal Península de Santa Elena. Seguimiento y Monitoreo a Resultados de Capacidad de Carga Turística a Balnearios de Santa Elena Escuela de Hotelería y Turismo. 